\title{
Editorials
}

\section{Artificial intelligence in primary care}

\section{INTRODUCTION}

Artificial intelligence (Al) in medicine captures the imagination with concepts such as the digital doctor, but also raises concerns such as replacing healthcare professionals, undermining trust in clinicians, and exacerbating inequalities. There is also continued uncertainty as to exactly what $\mathrm{Al}$ is. Given this confusion about definition, purpose, and potential, the RCGP has published an introductory report aimed at clarifying the position, ${ }^{1}$ in which a broad definition of Al as software with decision making capacity is used, ranging from software sequentially going through a series of yes/no questions (decision trees and algorithms) at the simplest, to complex software that learns from data (machine learning) where millions of datasets are used, for example, to find a pattern linking a set of symptoms and a disease.

Often, development of Al tools for placebased care and self-care is predominately driven by a focus on the technology and a commercial need to find a market, rather than the challenges and needs of healthcare professionals, practice and community providers, and patients. Unlocking the potential for Al means we must have healthcare professionals, patients, and technology experts working together and engaging with policy makers and commissioners.

\section{USE OF AI FOR CLINICAL DECISION- MAKING AND PROACTIVE DETECTION}

The development and provision of $\mathrm{Al}$ continues to progress rapidly. The Academic Health Science Network (AHSN), established by NHS England to spread innovation, released the results of a recent Al mapping survey, ${ }^{2}$ providing an overview of Al healthcare activity. While not exhaustive lorganisations including Babylon and Livi are missing), the survey provides some useful insights. Primary and community care Al clusters around two areas. First, clinical decision

"Al needs to be developed collaboratively with

\section{healthcare professionals and patients at all stages from idea to utilisation.}

making and care management, for example, symptom assessment, automating clinical coding, image recognition for dermatological conditions, triaging, and personalised selfmanagement. Second, proactive detection, such as, analysing patient records to predict patients with undiagnosed conditions such as dementia or belonging to vulnerable groups such as frail older people. Surprisingly, less than $10 \%$ of the survey responders are applying Al to reduce or automate administrative tasks.

\section{FRAMEWORKS SET THE FUTURE}

While there is both enthusiasm and scepticism about Al (sometimes expressed by the same individuals) we need to separate the hype from the reality - the use of Al will depend upon infrastructure, operating models, and data. 'The future of healthcare: our vision for digital, data and technology in health and care.3 is a policy paper published in October 2018 outlining a much needed approach to modernising healthcare computing systems, addressing current limitations in both infrastructure and operating models.

Data, on the other hand, can be a nebulous concept that continues to be a challenge. General practice records hold patient and practice information (or data) in various formats (codes, free text, letters) which can all be used to develop Al. However, biased, skewed, unrepresentative, and limited quantities of data affect Al functionality, potentially creating software with a higher likelihood of being erroneous and unsafe. Recent publication ${ }^{4,5}$ shine a light on the use and limitations of general practice data for narrow use cases: data need validating for each new application, which can be resource intensive. The use of patient data needs to be ethical from the perspective of patients, healthcare professionals, and the system, respecting choices on privacy, utilisation, access, and monetisation. Furthermore, these need to be mutually agreed, clearly communicated, and applied. Two recent publications aim to do just this; 'Data Ethics Framework.' complemented and built upon by Initial code of conduct for data-driven health and care technology.8 Together these set out the expectation the public sector holds on data use and transparency, providing reassurance when met. However, there are additional criteria related to the values held by primary care healthcare professionals which, if not met, could limit Al use. Continuity, evidence-based, overmedicalisation, safeguarding, anxiety induced demand, and management of uncertainty, are of high importance and could impede Al use if they are not incorporated and explained adequately.

'The future of healthcare," 'Data Ethics Framework,' and Code of Conduct,', set clear expectations, but it will take time to develop the guidance and mechanisms so that they are enforced and upheld, balancing self-policing and regulation of a technology that is mistrusted. In the meantime, the Al landscape risks being shaped by early established companies and decisions made with insufficient evaluations in place due to pressures to embrace technology. Al improves as more data becomes available; Al training is cumulative. It is easy to imagine the positive feedback loop: more data $=$ more accurate $\mathrm{Al}=$ better product $=$ more users = more data. Existing Al applications potentially have an advantage over newcomers; the gap widens with time producing monopolies and stifling competition.

Currently, Al needs to be developed for each use case (narrow Al) and retrained for different application areas, limiting lockin and monopolies. However, research is 
progressing to make Al transferable across applications.?

\section{THE NEW INTERFACE: CO-OPERATIVE AI AND THE HEALTHCARE PROFESSIONAL}

The concept of the professional working alongside a supportive system is not new. In manufacturing industries, attempts to replace people with robots and automation failed, and it was realised that robots have strength and are tireless, but they do not have the dexterity, agility, or flexibility of humans. This gave rise to collaborative robots, or cobots, a revised approach pairing the advantages of humans with the benefits of robotics, for example, automotive manufacturing cobots do highly repetitive tasks like screwdriving while human dexterity guides and facilitates assembly. Similarly, Al has advantages over humans in some areas while being much less capable in others - the true benefit is unlocked through combination working. Combined $\mathrm{Al} /$ human working augments the leadership, teamwork, creativity, social skills, observational skills, and empathy of healthcare professionals lor person accessing care), with the speed, scalability, information access, and quantitative capabilities of software. Research on 1500 organisations across sectors showed significant improvements from Al/human working. ${ }^{10} \mathrm{Al}$ tools that address unmet needs of patients and practice and community providers (for example, early detection of deterioration, automating communications, simplifying multi-login processes) will need a co-development approach with developers, healthcare professionals, provider organisations, patients, commissioners, and system level stakeholders as equal partners. Co-development is essential for Al to meet expectations and have acceptable limitations otherwise the bounds of function are the opinions of the developers, relying on quantitative data without qualitative data (preferences, context) with the risk of not necessarily being right.

Al has the potential to amplify human strengths, such as leadership and creativity, by providing relevant evidencebased information at the right time. ${ }^{10}$ Co-development leads to 'relevant' and 'right time' being defined in a way that meets the needs of healthcare professionals and of patients. Al can improve flexibility by creating chatbots which enable asynchronous one-to-many correspondences. With co-development, Al functionality and workflows embed the correct safeguards, have appropriate red flag functions, provide continuity, have acceptable clinical safety, avoids increasing anxiety, and have complementary processes supporting healthcare professionals. In an environment with insufficient numbers of healthcare professionals, Al can have a multiplying effect on available resources, such as automating identification of undiagnosed conditions or use digital systems to enable one-to-many remote monitoring. Through co-development we can achieve appropriate clinical oversight, safeguards, and management of risk.

To enable utilisation protocols, processes, workflows, and pathways will need altering to incorporate collaborative Al tools:

- healthcare professionals must have access to education to learn new skills as Al users and work differently;

- care providers using Al tools will need to be able to train Al systems to set them up to perform specified tasks. This means knowing what data to select and its quality;

- clinicians will need to be able to interpret Al outputs, which requires an understanding of its limitations and bounds of function; and

- users will need to know how the system learns and what constitutes appropriate use, so that ethical norms are upheld and any introduction of biases is avoided.

\section{CONCLUSION}

In future, some of this functionality will be built in, for example, self-selecting data to uphold original design principles, and commercial providers could offer to train an Al tool to a user's specification. Al needs to be developed collaboratively with healthcare professionals and patients at all stages from idea to utilisation. This will create trusted solutions to real problems for patient benefit. It will support acceptance by ensuring expectations on evidence, ethics, and the values of healthcare professionals are incorporated in evaluation methodology and regulation. Lastly it will support adoption by identifying the training required to change provider protocols, business processes, administrative workflows, and pathways. If developed collaboratively, Al can bring together the advantages of people and computers to minimise potential negative impact and to maximise health gain.

\section{Pritesh Mistry,}

Head of Innovation, Royal College of General

Practitioners, London.

\section{Provenance}

Commissioned; externally peer reviewed.

\section{Competing interests}

The author has declared no competing interests

\section{ADDRESS FOR CORRESPONDENCE}

\section{Pritesh Mistry}

Royal College of General Practitioners, 30 Euston Square, London NW1 2FB, UK.

\section{Email: Pritesh.Mistryarcgp.org.uk} amustbemistry

DOI: https://doi.org/10.3399/bjgp19X705137

\section{REFERENCES}

1. The Royal College of General Practitioners Artifical Intelligence and Primary Care. 2019. https://www.rcgp.org.uk/-/media/Files/CIRC/ artificial-intelligence-and-primary-carejan-2019.ashx?la=en laccessed 26 Jul 2019).

2. The Academic Health Science Network. Artificial Intelligence Mapping. 2019. http:// ai.ahsnnetwork.com/ai-mapping-2 laccessed 26 Jul 2019).

3. Department of Health \& Social Care. The future of healthcare: our vision for digital, data and technology in health and care. 2018. https://www.gov.uk/government/publications/ the-future-of-healthcare-our-vision-for-digitaldata-and-technology-in-health-and-care/ the-future-of-healthcare-our-vision-for-digitaldata-and-technology-in-health-and-care (accessed 26 Jul 2019).

4. Kostopoulou O, Sirota M, Round T, et al., The role of physicians' first impressions in the diagnosis of possible cancers without alarm symptoms. Med Decis Making 2017; 37(1): 9-16.

5. Hippisley-Cox J, Coupland C. Symptoms and risk factors to identify people with suspected cancer in primary care. Br J Gen Pract 2013; DOI: https://doi.org/10.3399/bjgp13X664135

6. Fenech M, Strukelj N, Buston O. Ethical, social, and political challenges of artificial intelligence in health. London: Future Advocacy \& Wellcome Trust, 2018. https://wellcome.ac.uk/ sites/default/files/ai-in-health-ethical-socialpolitical-challenges.pdf laccessed 26 Jul 2019).

7. Department for Digital, Culture, Media \& Sport. Data Ethics Framework. 2018. https://www. gov.uk/government/publications/data-ethicsframework/data-ethics-framework laccessed 26 Jul 2019).

8. Department of Health \& Social Care. Code of conduct for data-driven health and care technology. 2019. https://uww.gov.uk/ government/publications/code-of-conductfor-data-driven-health-and-care-technology/ initial-code-of-conduct-for-data-driven-healthand-care-technology (accessed 26 July 2019).

9. Dai W, Chen Y, Xue GR, et al. Translated learning: transfer learning across different feature spaces. California: Neural Information Processing Systems Foundation, 2008. https:// papers.nips.cc/paper/3492-translated-learningtransfer-learning-across-different-featurespaces.pdf (accessed 26 Jul 2019).

10. Wilson HJ, Daugherty PR. Collaborative Intelligence: Humans and Al Are Joining Forces. Harvard Business Review Press 2018; July-August: 114-123. 